\title{
Optical detection of single molecules in nanofluidic chips
}

\author{
Gea O.F. Parikesit ${ }^{* a}$, Vladimir G. Kutchoukov ${ }^{b}$, Wim van Oel ${ }^{\mathrm{a}}$, Guus L. Lung ${ }^{\mathrm{a}}$, \\ Andre Bossche $^{\mathrm{b}}$, Ian T. Young ${ }^{\mathrm{a}}$ and Yuval Garini ${ }^{\mathrm{a}}$ \\ Quantitative Imaging group ${ }^{\mathrm{a}}$, Electronic Instrumentation group ${ }^{\mathrm{b}}$, \\ Delft University of Technology, Lorentzweg 1, 2628 CJ Delft, The Netherlands.
}

\begin{abstract}
We present the latest progress on a novel technology for detecting and manipulating solution of single molecules in nanofluidic channels. This paper explains the design and fabrication of nanofluidic chip and its interface, molecule manipulation technique being used, and the optical detection method employed. Single molecule detections are performed using optical imaging as well as metal microelectrodes. The ultimate goal is to get high spatial and spectral resolutions that can lead to molecular identification.
\end{abstract}

Keywords: optical detection, nanofluidics, single molecules, lab on a chip.

\section{INTRODUCTION}

The study on single molecules has become more important in the recent years, particularly because it elucidates properties that are usually hidden in measurements on an ensemble. Detection and manipulation of single molecules are the keys in this study. One alternative approach is by employing nanofluidic structures. Liquid solutions containing single molecules are put into a flow through the nanoscale structures, such that detection and manipulation can be done on each single molecule. Microelectrodes are employed to perform electrokinetic manipulation on the single molecules. Optical detection is used due to its high sensitivity, its ability to detect multiple wavelengths, and the abundance of optical methods that can be used on the same chip without modifying it. This should lead to the high resolution, spatially and spectrally, in the detection system.

We will start with a brief explanation on the manipulation method that will be used in the nanofluidic chip, which could enable separation of single molecules according to their charge and polarizability. Section 3 describes the design and the fabrication method of the nanofluidic chips. A versatile interface made for connecting the nanofluidic chip to the outerworld instruments is discussed in section 4 . Section 5 provides the results of first observation on one of the nanofluidic chips. Afterwards, section 6 gives the conclusion of this paper.

\section{MOLECULE MANIPULATION METHOD}

\subsection{Acting forces in the nanofluidic chip}

Three different electrokinetic forces can be applied on the solution of molecules or particles inside our nanofluidic chip: electroosmosis, electrophoresis, and dielectrophoresis. In the case of electroosmosis, as a liquid fills a nanofluidic channel, an electric double layer will be formed in the interface between the liquid and the channel's wall ${ }^{1,2}$. This double layer comprises the negative ions of the wall and positive ions of the liquid. If the inlet of the channels is connected to a positive electrode, while the outlet is connected to a negative electrode, then the liquid's positive ions in the double layer will move to the outlet. Due to the viscosity of the liquid, the bulk liquid is also dragged along in the same direction. The liquid moves with a velocity of

$$
\mathrm{v}_{\mathrm{EO}}=\frac{\varepsilon_{0} \varepsilon_{l} \zeta}{\eta} \mathrm{E}
$$

\footnotetext{
gea@qi.tnw.tudelft.nl; phone: +31 15278 6054; fax +31 152786740
} 
where $\varepsilon_{0}$ is the vacuum permittivity, $\varepsilon_{1}$ is the relative permittivity of the liquid, $\zeta$ is the electric potential in the double layer, $\eta$ is the fluid viscosity, and $E$ is the electric field vector. Therefore the electroosmotic force is given as

$$
\mathrm{F}_{\mathrm{EO}}=m_{l} \frac{d \mathrm{v}_{\mathrm{EO}}}{d t},
$$

where $m_{l}$ is the mass of the bulk liquid.

Meanwhile in the case of electrophoresis, particles inside the liquid solution also interact with the electrodes set in the inlet and outlet of the channels ${ }^{1,2}$. Should the particles have no charge, they will move along with the liquid, which is driven by electroosmosis. If the density of the particles is not much different than the liquid's density, then the liquid and the particles have approximately the same direction and velocity. However, if the particles are charged, the velocity of the particles and the liquid can be different. Positively charged particles will feel an attraction force toward the outlet, so they move faster than the liquid. Meanwhile, negatively charged particles will feel an attraction force toward the inlet, such that their movement toward the outlet is slower than the liquid. The attraction force on the charged particles is

$$
\mathrm{F}_{\mathrm{EP}}=q_{p} \mathrm{E},
$$

with $q_{p}$ as the charge of the particle.

Dielectrophoresis is the third acting electrokinetic force. As neutral polarizable particles are put in a non-uniform electrical field, each of them will be polarized and form a dipole. The two poles interact with the electric field, and are attracted to different directions with unequal force magnitude. Therefore, the particles will move toward the region with highest electric field regardless to the orientation of the electric field ${ }^{1,2}$. Thus AC as well DC electric field can be used. This is the so-called positive dielectrophoresis. However, if the particles are less polarizable than the liquid, they will experience lagging in aligning their dipoles to the electric field. In this case, electric field will be distorted around the particles, and the induced dipole will have opposite orientation. Hence the particles will move toward the region with lowest electric field, experiencing the so-called negative dielectrophoresis. The dielectrophoresis force is expressed as

$$
\mathrm{F}_{\mathrm{DEP}}=\frac{1}{2} \operatorname{Re}\left\{\left[4 \pi \varepsilon_{l} r_{p}^{3} K(\omega) \mathrm{E}\right] \cdot\left[\nabla \mathrm{E}^{*}\right]\right\},
$$

where $r_{p}$ is the particles' radius, $\omega$ is the electric field frequency, and $K(\omega)$ is the Claussius-Moratti factor described by

$$
K(\omega)=\frac{\varepsilon_{p}^{\prime}-\varepsilon_{l}^{\prime}}{\varepsilon_{p}^{\prime}+2 \varepsilon_{l}^{\prime}},
$$

with $\dot{\varepsilon}_{p}$ and $\dot{\varepsilon}_{l}$ the complex permittivities of the particles and the liquid, respectively. When $\operatorname{Re}\{K(\omega)\}$ is positive, the particles experience positive dielectrophoresis, while a negative $\operatorname{Re}\{K(\omega)\}$ leads to negative dielectrophoresis. Therefore, different $\omega$ could lead to different types of dielectrophoresis acting on a certain particle.

Besides the three electrokinetic forces described above, there is still the hydrodynamic force. This force exists only if pressure difference is applied between the ends of the fluidic channel. In this case, Stokes drag on the particle results in the following force:

$$
\mathrm{F}_{\mathrm{HD}}=\left(\mathrm{v}_{\mathrm{p}}-\mathrm{v}_{1}\right) 6 \pi r_{p} \eta
$$

with $r_{p}$ as the particle's radius, and $v_{p}$ and $v_{l}$ as the velocities of the particle and the liquid, respectively.

\subsection{Concept of the separation method}

In this research, several nanofluidic structures have been designed. One of the designs is shown in Figure 1, in top-view. The inlet and outlet can be seen from the arrows in Figure 1(a). The height of all the nanofluidic structures is $150 \mathrm{~nm}$, much less than its width or length. Therefore, the two-dimensional laminar flow assumption can be applied. Moreover, possibility for several molecules positioned on top of each other is reduced.

After passing a straight channel $100 \mu \mathrm{m}$ wide, the liquid solution enters a half-circle chamber with a diameter of $1 \mathrm{~mm}$. From the chamber, the particles can exit through one of the twelve $100 \mu \mathrm{m}$-wide sub-outlets available. In these chips, those sub-outlets are combined to a $273 \mu \mathrm{m}$-wide outlet, but in a final version each of the sub-outlets can lead to a 
different way. At the left-upper and right-upper corners of Figure 1(a), two separated electrodes were made, encircling the half-circle chamber. A photograph of the fabricated nanofluidic structure is shown in Figure 1(b).

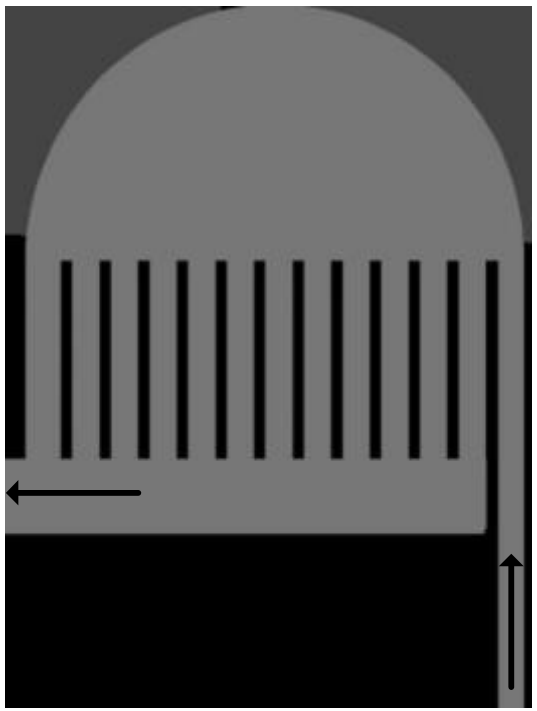

(a)

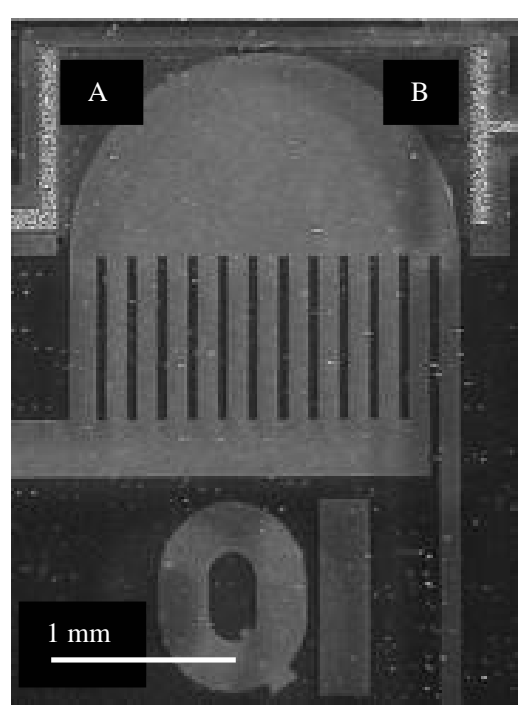

(b)

Figure 1. One of the nanofluidic structure designs (in top-view).

(a) The schematic design, with arrows showing the incoming and outgoing direction of flow.

(b) A photograph of the fabricated structure. A and B show the two microelectrodes used for dielectrophoresis.

The white scalebar represents $1 \mathrm{~mm}$.

The nanofluidic structure illustrated above was fabricated to perform separation of molecules or particles based on their physical properties. Once the liquid solution goes inside the structure, positive and negative voltages can be applied to the inlet and the outlet, respectively. Therefore the liquid will move from the inlet to the outlet, experiencing electroosmosis. Charged particles will move either faster or slower than the liquid, due to electrophoresis.

Meanwhile, the two microelectrodes around the half-circle wall are designed for dielectrophoresis. AC voltages applied to them will induce an AC electric field inside the structure, as illustrated in Figure 2(a). AC, instead of DC voltages, are chosen so that the two microelectrodes will not induce unwanted electrophoresis. Along the half-circle wall, magnitude of the electric field is the highest. Polarizable neutral particles will be deflected by the non-uniform field and experiencing dielectrophoresis. Therefore particles more polarizable than the liquid will be attracted toward the halfcircle wall and their path will get longer. On the other hand, particles less polarizable than the liquid will be repelled from the half-circle wall and their path will be shorter. The shorter paths lead to the sub-outlets nearer to the inlet, while the longer paths lead to the sub-outlets further away from the inlet.

In this structure design, hydrodynamic pressure differences will not be introduced between the inlet and the outlet. Eventually, molecules or particles with different charges, and polarizations will travel in different paths and velocities inside the structure, as illustrated in Table 1 and Figure 2(b).

Table 1: Different charge and polarizability will lead to different paths and velocities inside the nanofluidic structure.

\begin{tabular}{|c|c|c|c|}
\hline \multirow{2}{*}{ Neutral } & Particle properties & Path length & Velocity \\
\cline { 2 - 4 } & Not easily polarized (Neg. DEP) & Long & Normal \\
\hline \multirow{2}{*}{ Charged } & Positively charged & Normal & Normal \\
\cline { 2 - 4 } & Negatively charged & Normal & Slow \\
\hline
\end{tabular}




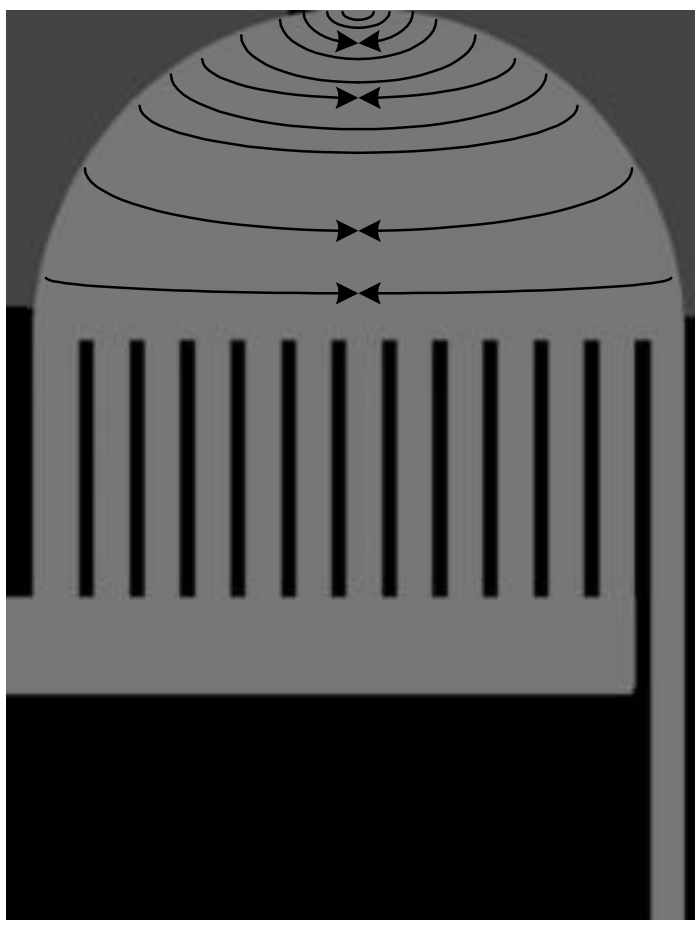

(a)

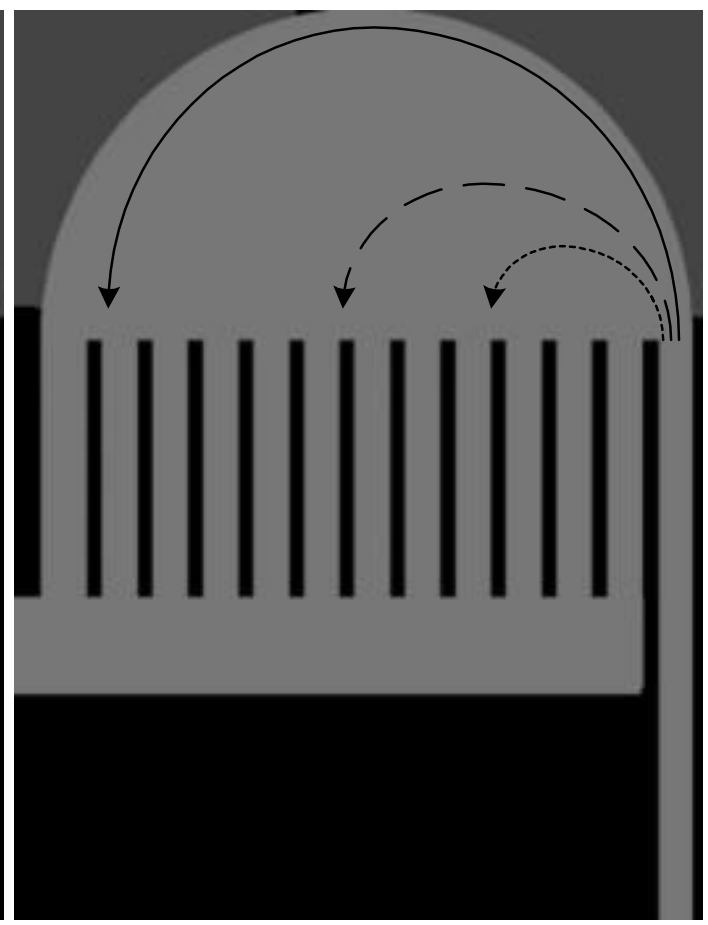

(b)

Figure 2. Electrokinetic manipulation and separation inside the nanofluidic structure.

(a) AC electric field between the dielectrophoresis electrodes.

(b) Travelling path of charged particles (dashed line), neutral particles with positive dielectrophoresis (straight line), and neutral particles with negative dielectrophoresis (dotted line).

\section{CHIP FABRICATION}

Two types of nanofluidic chips were fabricated for this study. The first type uses only doped a:Si (amorphous silicon) as electrodes, while the second type also employs Al (aluminum) to improve the conductance of the electrodes. Both types of chips are fabricated simultaneously in a single wafer, thus the fabrication method is convenient.

The electrodes are self-aligned. This means the height of the electrodes and the nanofluidic channels are alike, thus no fluid leakage would occur. In the first type of the chip, the a:Si layer that is used as an electrode layer is doped with As+ (arsenic ion). These dopants are activated using excimer laser annealing, with a wavelength of $309 \mathrm{~nm}$. To avoid doping on unwanted regions, a protective layer comprising oxide and metal was used to mask them; this protective layer was removed afterwards. The silicon absorbs deep-UV strongly, and heat is generated only in the a:Si layer. In that way, the temperature of the substrate is kept low and the glass does not soften. The fabrication starts by depositing $33 \mathrm{~nm}$ a:Si which is required for the bonding. As+ was implanted and activated to make the electrodes. Afterwards some undoped a:Si around the doped a:Si were removed, to avoid current leakage from the doped a:Si. Finally, anodic bonding was performed, where the capping wafer has pre-drilled holes for nanofluidic channel's inlet/outlet and for access to the doped a:Si electrodes. The sheet resistance of these doped a:Si electrodes was measured as $432 \mathrm{Ohm} / \mathrm{square}^{3}$.

To overcome the low conductance of the doped a:Si electrode, a second type of chips were fabricated. In these chips, $\mathrm{Al}$ was used as electrodes, until very near the nanofluidic channels. To avoid unwanted chemical reaction between the liquid solution and non-noble metal, $\mathrm{Al}$ should not directly touch the liquid solution inside the channels. Therefore, doped a:Si electrodes are used in between the end of the $\mathrm{Al}$ electrodes and the nanofluidic channels. This also helps in maintaining the planarity of the structure. To ensure that the bonding is properly formed, the Al metal were deposited in grooves so that their height does not exceed the nanofluidic channel's height. Fabrication began similarly like the first type of chips, except that some more glass and a:Si layers were removed to make the groove for the Al. The As+ was also implanted and activated, and some undoped a:Si around the doped a:Si were also removed. Afterwards, Al was deposited inside the 
groove. As the final step, anodic bonding was performed similarly like in the first type of chips. This time, the access is to the $\mathrm{Al}$ electrodes, which is over the doped a:Si electrodes. To test the resistance of the electrodes, we performed measurement of the sheet resistance as well. The result is $0.044 \mathrm{Ohm} /$ square. Thus the conductance of the $\mathrm{Al}$ electrodes is approximately 10,000 times better than the conductance of the doped a:Si electrodes. The schematic of the fabrication steps are shown in Figure 3, for the first and second types of chips. In both cases, the doped a:Si touches the edge of the nanofluidic channel.

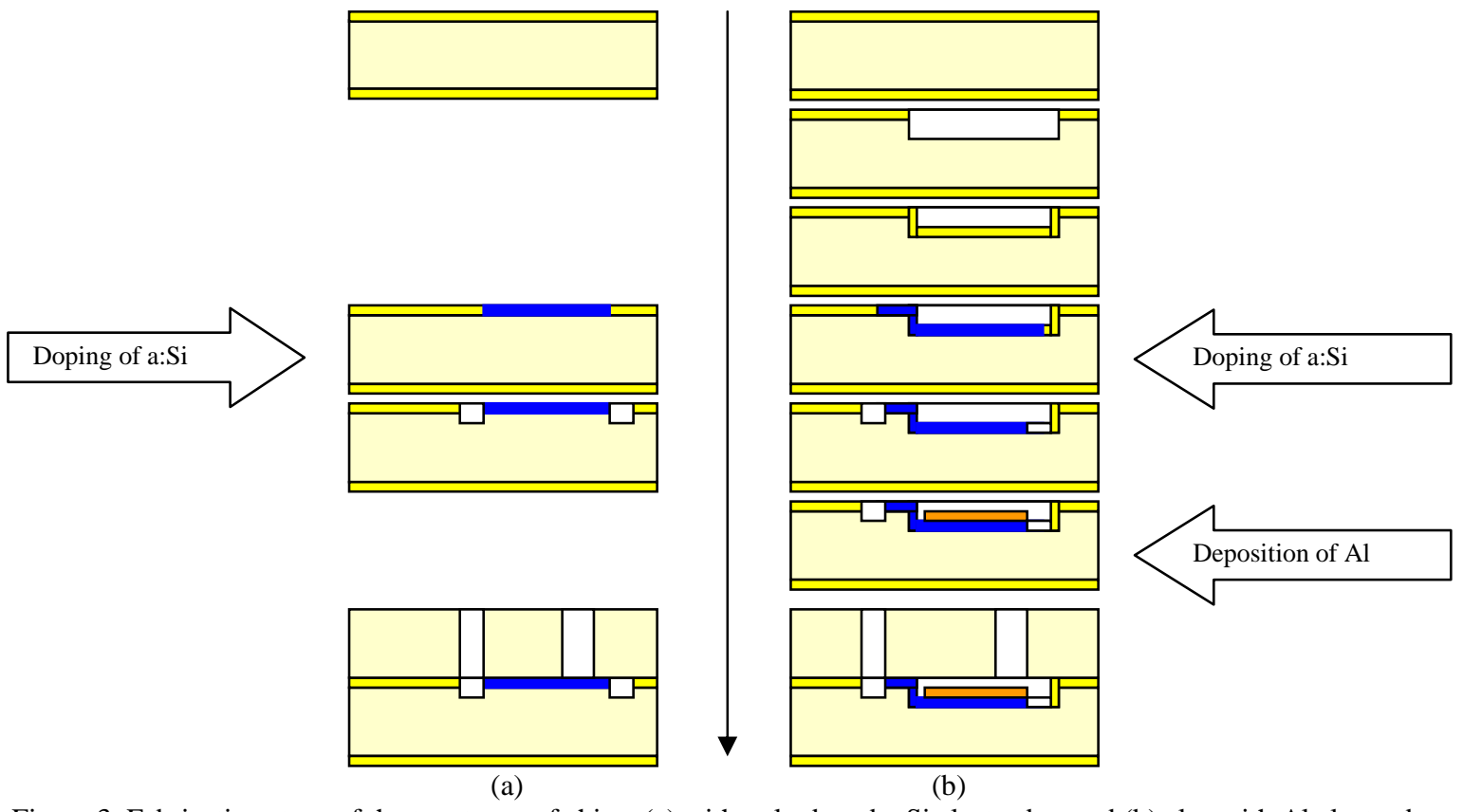

Figure 3. Fabrication steps of the two types of chips: (a) with only doped a:Si electrodes, and (b) also with Al electrodes.

\section{INTERFACE}

Stable fluidic and electronic connections are required between the nanofluidic chip's features (channels' inlet/outlet, doped a:Si electrodes, and Al electrodes) and the outer-world instruments (fluid dispensers, fluid reservoirs, voltage sources, voltmeters, etc). To provide this, we developed an interface from Perspex plastic with a dimension of $76 \times 40 \times 20$ $\mathrm{mm}^{3}$ (see Figure 4).

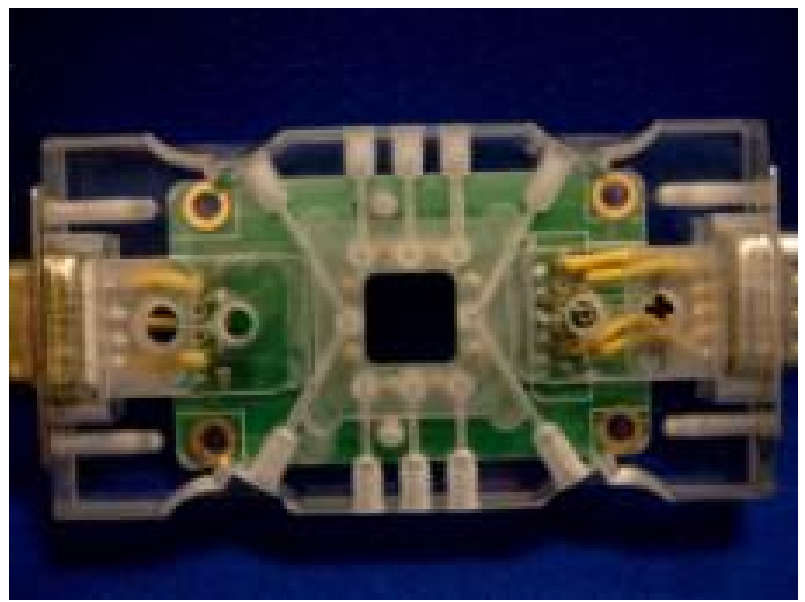

(a)

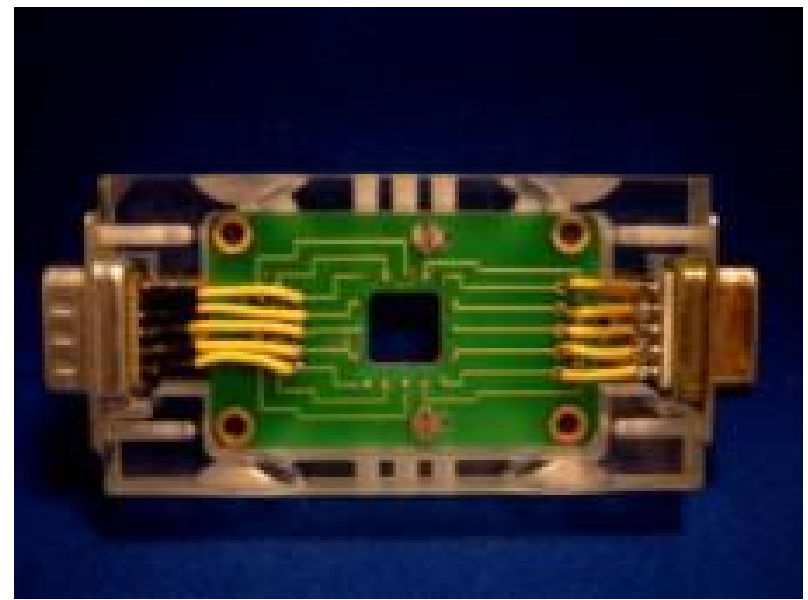

(b)

Figure 4. Top view (a) and bottom view (b) of the interface. 
The interface was designed so that when the chip is held firmly in this interface, fluidic and electronic features can be controlled from outer-world instruments with ease. The fluidic ports comprise six normal-ports and two pairs of flushingports; each port can be used as an inlet or outlet. When one part of the flushing-ports is opened, the ports act similarly like the normal-ports; however, when both parts of the flushing ports are opened, liquid solution are flushed and can be changed quickly from one type to another. To connect the fluidic ports to fluid dispensers/reservoirs, microfluidic parts from Upchurch are used. All these fluidic ports are also equipped with gold wires for electrokinetic manipulation.

For the electronic ports, ultra-mini pogo pins from Emulation Technology are employed. These pins ensure firm connections between electrodes in the chip and wires in the interface. At the bottom part of the interface, a printedcircuit-board is attached, and it connects both the pogo-pins and fluidic ports' gold wires to outer-world cable connections. In total, the board organizes eight pogo-pins and eight gold wires, and is designed to allow voltages up to 500 Volts.

On the top part of the interface, a 20x20 $\mathrm{mm}^{2}$ groove was made to hold the nanofluidic chip. A window hole was mode through the center part of the interface from top to bottom, so that this versatile interface can be used with both transmission and reflection microscopes.

\section{FIRST OBSERVATION}

The detection of the molecules or particles inside the nanofluidic chip can be done using some microscopy techniques, among others the fluorescence microscopy ${ }^{4}$. However, as an alternative to overcome bleaching that can happen in with fluorescence beads, metallic PRP (plasmon resonant particles) can also be used ${ }^{5}$. Either PRP or fluorescence beads can be attached to single biological molecules, so that the molecules' dynamics can be studied. Spectral imaging ${ }^{6}$ can $^{2}$ also be further applied, so that molecules emitting different wavelengths can be detected and identified simultaneously.

First observation was done using fluorescence microscopy, on the region illustrated by the white rectangle in Figure 5. A solution of fluorescence beads (with a diameter of $20 \mathrm{~nm}$ and emission wavelength of $515 \mathrm{~nm}$ ) was flowing through the nanofluidic structure shown in Figure 1(b). A Hamamatsu CCD camera, mounted on a Leica confocal microscope, captured the images in 15 frames per second. A voltage difference of 150 Volts was applied between the inlet and the outlet, to introduce electrophoresis. The microelectrodes for dielectrophoresis were not used yet. No pressure difference between the inlet and the outlet was applied. Some parts of the observation results are shown in Figure 6 and 7.

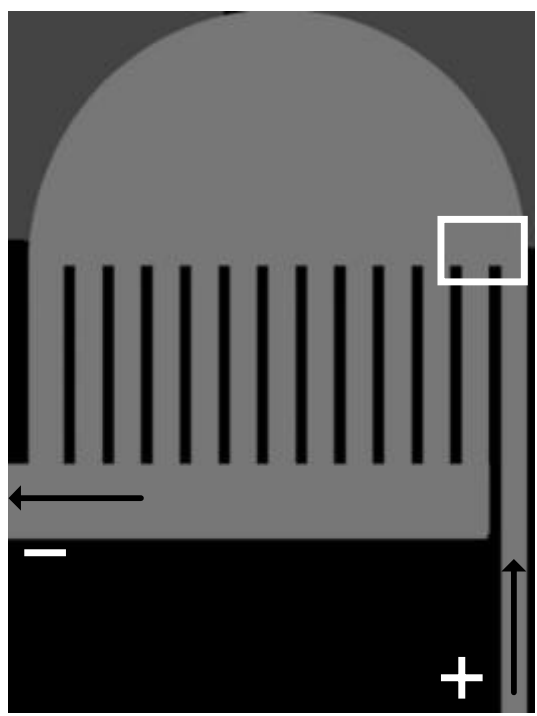

Figure 5. The region of observation, as shown with the white rectangle. 


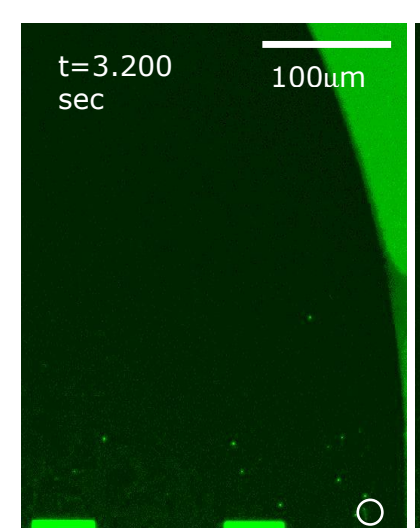

(a)

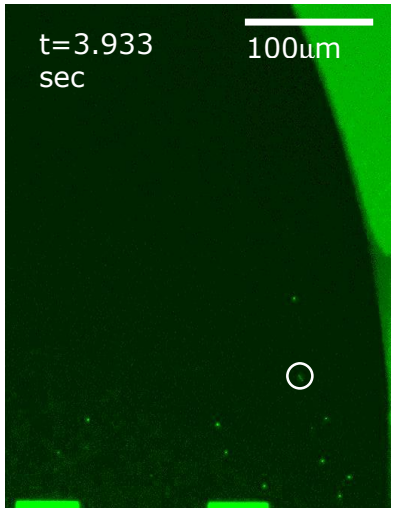

(b)

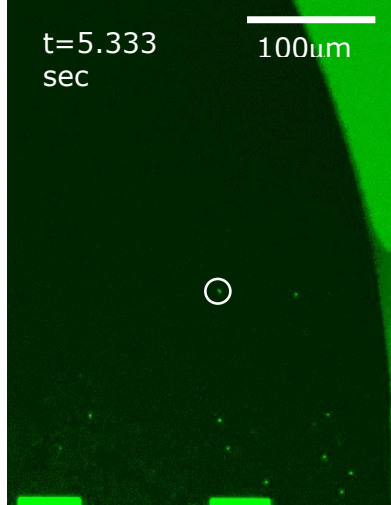

(c)

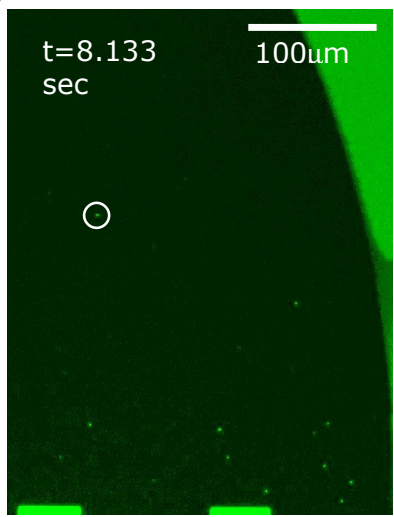

(d)

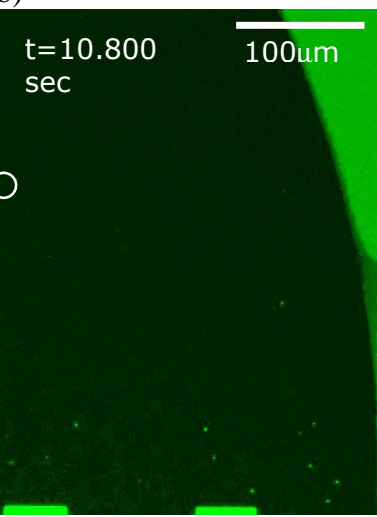

(e)

Figure 6. A fluorescence bead (marked with a white circle) flows into the half-circle chamber.

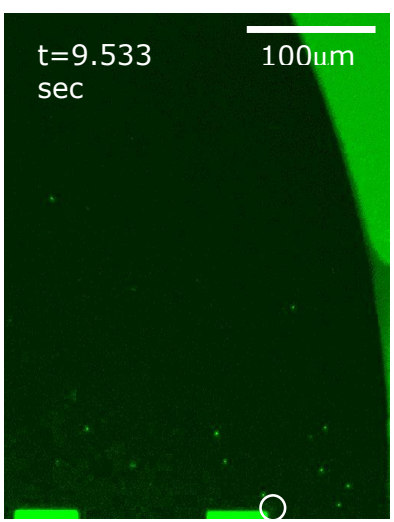

(a)

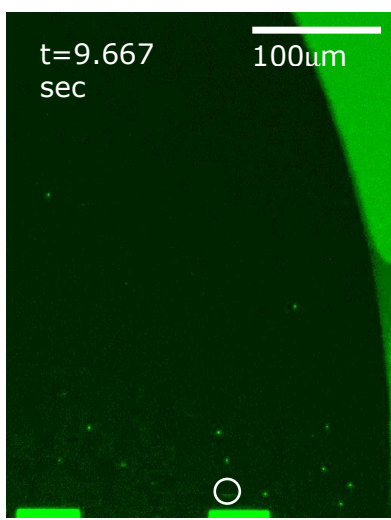

(b)

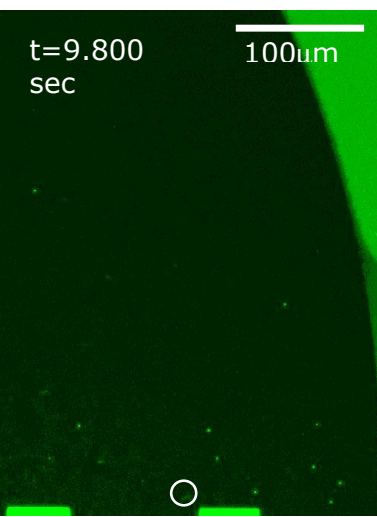

(c)

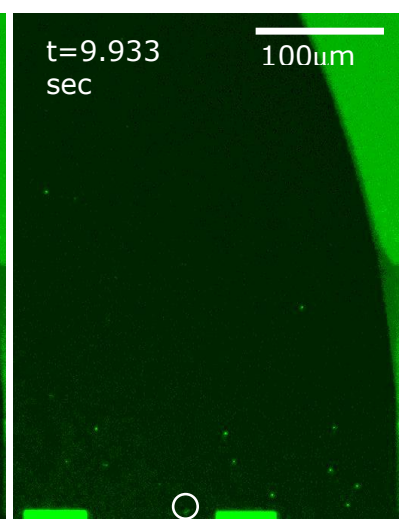

(d)

Figure 7. A fluorescence bead (marked with a white circle) makes a sharp turn into the nearest sub-outlet.

Figure 6 shows one bead flowing from the inlet into the half-circle chamber, while Figure 7 shows another bead making a sharp u-turn from the inlet into the nearest sub-outlet. The bead in Figure 6 experienced a fast motion when it starts coming from the inlet. It gets slower as it reaches the center of the half-circle chamber. Meanwhile, the bead shown in Figure 7 made a very fast u-turn. The trajectories and the varying velocities of those flowing beads are according to the expected laminar flow. Since the beads' mass is so small, no significant inertia effect occurs. All the beads just follow the fluid flow, acting as tracers, driven by electroosmosis. However, as seen both in Figures 6 and 7, some beads were observed as not moving at all. Those are the bigger beads, which results from the non-uniformity of the fluorescence beads employed; filtering of the solution prior entering the nanofluidic chip could have eliminated those bigger beads. 
We observed no beads going in-focus or out-of focus during the flow. That means the whole nanofluidic channel's height is within the microscope's depth-of-field. No two beads were seen positioned on top of each other, thus the channel's height is small enough to validate the two-dimensional flow assumption.

\section{CONCLUSION}

We have presented a novel nanofluidic technology that would enable manipulation and separation of single molecules using electrokinetic methods. Detection and identification of the single molecules can be done using various microscopy techniques, among others the fluorescence microscopy. We also presented the fabrication steps of the nanofluidic chips and described the interface that connects the chips to outer-world instruments. First observations on fluorescence beads flowing in one of the nanofluidic chips, driven by electroosmosis, shows that the expected two-dimensional laminar flow assumption holds. Further research will be done to prove and develop the concept of the separation method proposed in section 2.2 in this paper.

\section{ACKNOWLEDGEMENTS}

This research is supported by the Dutch Foundation for Fundamental Research on Matter (FOM).

\section{REFERENCES}

1. N. Nguyen, S. Wereley, Fundamentals and Applications of Microfluidics, Artech House, 2002.

2. M.P. Hughes, Nanoelectromechanics in Engineering and Biology, CRC Press, 2003.

3. V.G. Kutchoukov, L. Pakula, G. O. F. Parikesit, Y. Garini, L. K. Nanver, A. Bossche, Nanochannels in Glass with Poly-Silicon Electrodes, Proceedings of Eurosensors, 2004, to be published.

4. Ch. Zander, J. Enderlein, R.A. Keller (eds) Single Molecule Detection in Solution - Methods and Applications, Wiley VCH, 2002.

5. S. Schultz, D.R. Smith, J.J. Mock, D.A. Schultz, Single-target molecule detection with nonbleaching multicolor optical immunolabels, PNAS, Vol.97, No.3, pp. 996-1001, 2000.

6. R.M. Levenson, C.C. Hoyt, Spectral imaging and microscopy, American Laboratory, 2000. 